\title{
Martensitic transition and structural modulations in the Heusler alloy $\mathrm{Ni}_{2} \mathrm{FeGa}$
}

\author{
J.Q. Li* ${ }^{*}$ Z.H. Liu, H.C. Yu, M. Zhang, Y.Q. Zhou, G.H. Wu \\ Institute of Physics, Chinese Academy of Sciences, Beijing 100080, People's Republic of China
}

Received 9 February 2003; accepted 20 February 2003 by H. von Löhneysen

\begin{abstract}
We have found two distinctive structural modulations altering evidently along with the martensitic transition (MT) in the

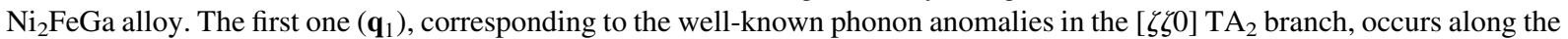
$\langle 110\rangle$ direction. The second one $\left(\mathbf{q}_{2}\right)$, an incommensurate modulation observed for the first time, occurs along the $\langle 211\rangle$ direction. Both modulations change gradually with the premartensitic phonon softening and discontinuously with the MT. Anomalies in magnetic properties emerging around the MT have been briefly discussed.

(C) 2003 Elsevier Science Ltd. All rights reserved.
\end{abstract}

PACS: 64.70.Kb; 75.50.Gg; 61.14.Lj; 61.44.Fw

Keywords: A. Magnetically ordered materials; C. Scanning and transmission electron microscopy; D. Phase transition

The martensitic phase transformations in a rich variety of ferromagnetic shape-memory alloys have been extensively investigated in past years [1-5]. Among the large number of ferromagnetic Heusler alloys, the intermetallic $\mathrm{Ni}-\mathrm{Mn}-\mathrm{Ga}$ alloy is a typical one undergoing a martensitic transition (MT) at $T_{\mathrm{m}} \sim 200 \mathrm{~K}$, which, in our knowledge, could give rise to the maximum magnetic-field-induced strain [5-10]. In this kind of materials, experimental and theoretical investigations have been specially focused on the anomalies behavior in the [110]-TA 2 phonon branch [11], evident structural distortions, corresponding to atomic displacements along $\langle-110\rangle$ direction, was identified in a large temperature range $[11,12]$. Very recently, a new Heusler alloy with the nominal composition of $\mathrm{Ni}_{2} \mathrm{FeGa}$ has been successfully synthesized using melt-spinning technique in our lab [13]. Structural analyses clearly indicate that $\mathrm{Ni}_{2} \mathrm{FeGa}$ ribbons contain a well-defined $\mathrm{L} 2{ }_{1}$ order at room temperature. The $\mathrm{Ni}_{2} \mathrm{FeGa}$ alloy undergoes a ferromagnetic transition at around $T_{\mathrm{c}} \sim 430 \mathrm{~K}$ and a MT at around $145 \mathrm{~K}$, upon this MT transition, the $\mathrm{Ni}_{2} \mathrm{FeGa}$ materials yield a

\footnotetext{
* Corresponding author. Tel.: +86-1082649524; fax: + 86 1082649531.

E-mail address: ljq@aphy.iphy.ac.cn (J.Q. Li).
}

completely recoverable two-way shape memory effect. Principally, the field-induced strain occurring in ferromagnetic shape-memory alloys is due to the influence of the field on the structural variants of martensite [11,14]. Hence, a careful structural analysis, especially the evolution of microstructure along with the MT, should play an important role for understanding the significant properties of this new material. In the present study, the structural properties of the $\mathrm{Ni}_{2} \mathrm{FeGa}$ Heusler phase have been investigated in the temperature range of $100-1100 \mathrm{~K}$. In particular, structural modulations, MT and related anomalies in physical properties have been extensively analyzed.

In our study, alloy samples of $\mathrm{Ni}_{2} \mathrm{FeGa}$ were prepared by melting pure metals in proportion in an induction furnace under the argon atmosphere. Experimental details on the sample synthesis have been reported in Ref. [13]. Some samples are further annealed in a vacuum environment $\left(\sim 10^{-3} \mathrm{~Pa}\right)$ for eliminating certain metastable structural distortions. Specimens for transmission-electron-microscope (TEM) observations were polished mechanically to a thickness of around $30 \mu \mathrm{m}$, dipped to $20 \mu \mathrm{m}$, and then ionmilled. The structural investigations were performed on a H-9000NA TEM equipped with the cooling and heating sample stages. 
Fig. 1 shows the electron-diffraction patterns exhibiting the notable structural features of the $\mathrm{Ni}_{2} \mathrm{FeGa}$ alloy at room temperature. These patterns are taken with electron beam slightly off the [100] zone-axis direction for Fig. 1(a), and along the [011] direction for Fig. 1(b). The main diffraction spots in both patterns can be well indexed by a cubic cell with lattice parameter of $a=5.74 \AA$. Appearance of the sharp (111) diffraction spot in Fig. 1(b) directly suggests a well-defined L2 1 chemical order (Heusler phase) in current system [13-15]. The most strike features revealed in our observations are the presence of additional reflection spots and streaks following the main diffractions. These diffuse satellite spots, corresponding to micro-modulated structures, emanate from each of the Bragg diffraction spot along both the $\langle 110\rangle$ and $\langle 211\rangle$-directions. These features, for instance, can be clearly recognized around the (040) spot in Fig. 1(a) and (822) spot in Fig. 1(b). In the previous studies of $\mathrm{Ni}-\mathrm{Mn}-\mathrm{Ga}$ and $\mathrm{Ni}_{x} \mathrm{Al}_{100-x}$ alloys, the diffuse diffractions along $\langle 110\rangle$ direction are discussed in connection with premartensitic transition and phonon anomalies in the [ $\zeta \zeta 0]$ $\mathrm{TA}_{2}$ branch of the Heusler phase. Structural anomaly along the $\langle 211\rangle$-direction is a new feature appearing in the Heusler phase $\mathrm{Ni}_{2} \mathrm{FeGa}$. In our investigations, we found that both modulations alter systematically with low-temperature phase transitions in $\mathrm{Ni}_{2} \mathrm{FeGa}$.

Structural modulation along the $\langle 110\rangle$ direction $\left(\mathbf{q}_{1}\right)$, as mentioned above, can be essentially understood by the softening of the [110] $\mathrm{TA}_{2}$ phonon branch. Atomic displacements are along $\langle-110\rangle$ direction, resulting in a typical transverse polarization. The experimental studies suggest that its maximum intensity occurs at $(1 / 3,1 / 3,0)$ position as indicated by arrows in Fig. 1(a). Microstructural properties corresponding to this kind of modulation
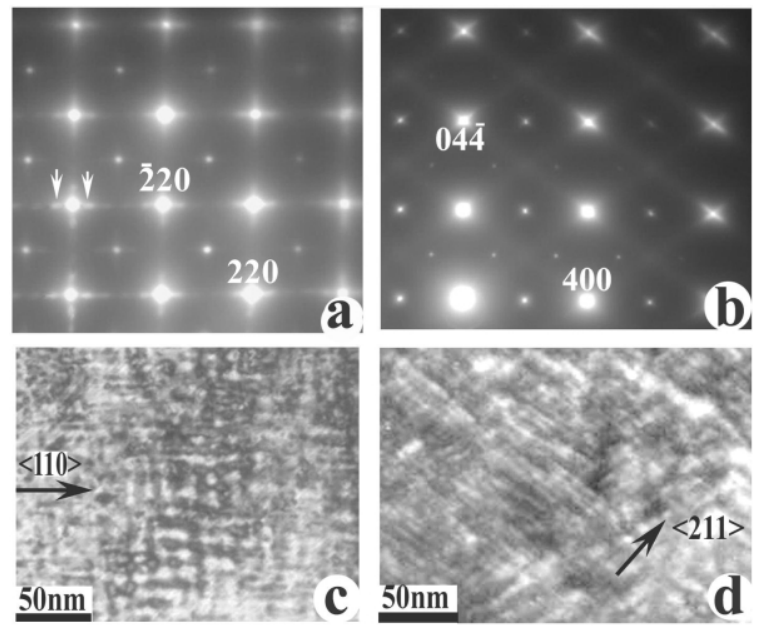

Fig. 1. Electron diffraction pattern taken (a) slightly off the [100] and (b) along the [011] zone axis directions, exhibiting the additional reflection spots and streaks. (c) TEM image showing the tweed pattern in the $a-b$ plane. (d) TEM image showing the micro-domain lamellas within the (011) plane. can be well characterized by the tweed pattern. Fig. 1(c) shows a dark-field TEM image taken by using the (400) spot and its following satellite streaks. Similar pattern has also observed and interpreted in the study of $\mathrm{Ni}-$ $\mathrm{Mn}-\mathrm{Ga}$ alloy [12]. Structural modulation along the $\langle 211\rangle$ direction $\left(\mathbf{q}_{2}\right)$ gives rise to another kind of micro-domain pattern within the (011) crystal plane. Fig. 1(d) shows a dark-field TEM image taken by (422) spots and its satellite streaks, illustrating the presence of micro-lamella pattern along the $\langle 211\rangle$ and $\langle-211\rangle$ directions.

The MT in present system occurs at the critical temperature $T_{\mathrm{m}}=145 \mathrm{~K}$. Our TEM observation directly suggests that both modulations show up remarkable changes along with this transition. Above the martensitic critical point $\left(T_{\mathrm{m}}\right)$, the diffuse satellites change progressively toward superstructure spots with lowering temperature, and below $T_{\mathrm{m}}(145 \mathrm{~K})$, satellite reflections turn out to be sharp spots corresponding to several typical superstructures. Fig. 2(a) shows a typical superstructure appearing in the basic crystal plane taken at $100 \mathrm{~K}$. This superstructure appears along the $\langle 110\rangle$-direction with a wave vector of $\left(2 a^{*}+2 b^{*}\right) / 6$, i.e. a wavelength of six-times the distance between $\{220\}$ planes. Conventionally, it is called
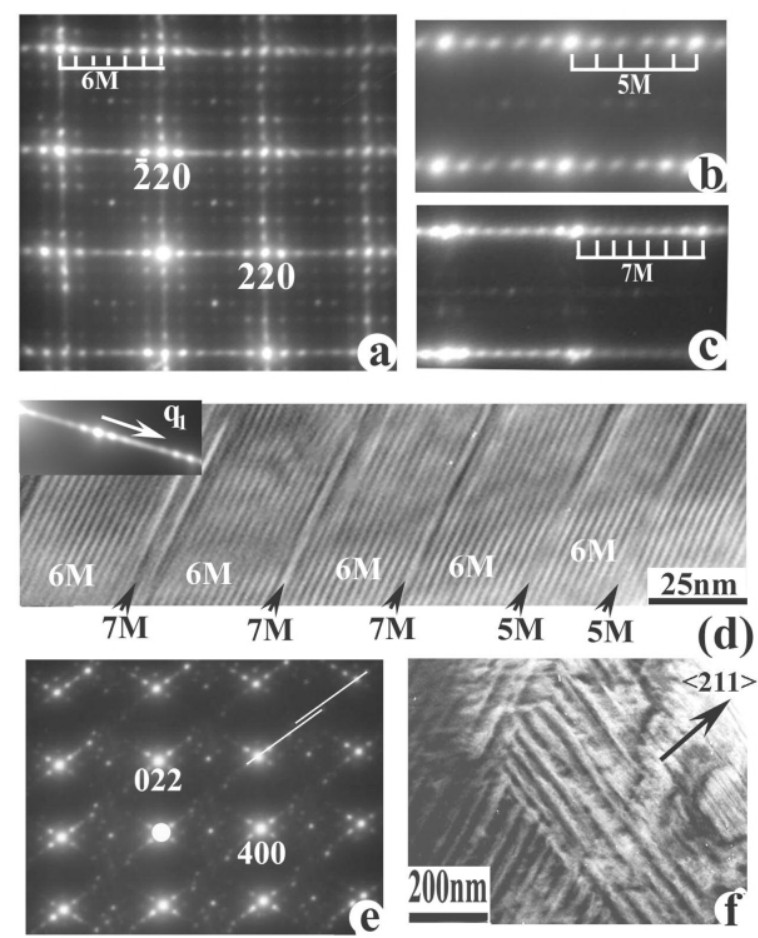

Fig. 2. Electron diffraction patterns taken at low temperature of $100 \mathrm{~K}$, showing the presence of superstructures along $\langle 110\rangle$ direction: (a) 6M, (b) 5M, and (c) 7M. (d) TEM image showing the intergrowth lamellas of the $5 \mathrm{M}, 6 \mathrm{M}$, and $7 \mathrm{M}$ superstructures. (e) The electron-diffraction pattern showing the strong incommensurate modulation slightly off the $\langle 211\rangle$ direction. (f) The low-temperature domain structure within the (110) plane. 
martensite $6 \mathrm{M}$ superstructure. This observation is well consistent with the theoretical investigations suggesting a soft condensation towards the $(1 / 3,1 / 3,0)$ mode. In this pattern, two perpendicular sets of satellite reflections are clearly visible around each basic Bragg spot. Those are considered to originate from twin domains where the modulation wave vectors are rotated by $90^{\circ}$ with respect to one another. Careful observation also suggest the evolution of the $\mathbf{q}_{1}$ modulation is strongly affected by sublattice during the MT, as a result, two weak superstructures with the respective wavelengths of five- and seven times the distance between $\{220\}$ planes appear frequently following with the $6 \mathrm{M}$ superstructure. Fig. 2(b) and (c) shows two nano-diffraction patterns illustrating the existences of $5 \mathrm{M}$ and $7 \mathrm{M}$ superstructures in small areas. Highresolution TEM observations frequently reveal the presence of intergrowth lamellas of different superstructures. Fig. 2(d) shows a low temperature TEM image illustrating this kind of inter-growth lamellas from $5 \mathrm{M}, 6 \mathrm{M}$ and $7 \mathrm{M}$ superstructures in an area. The diffraction pattern from this area is also displayed in the inset. Another kind of remarkable structural feature in the low-temperature martensite is the appearance of an incommensurate modulation along the $\langle 211\rangle$ direction, which is believed to be in association with the phonon anomalies in the $[2 \eta, \eta, \eta]$ branch. Fig. 2(e) shows a typical electron diffraction pattern revealing such an incommensurate modulation at the temperature of $100 \mathrm{~K}$. Actually, this modulation propagates slightly off the $\langle 211\rangle$ direction, the satellite-spot sequences belonging to adjacent rows of main spots exhibit both spacing and orientation anomalies: no single straight line can pass through the satellite sequences in adjacent bands. The modulation wave vector can be roughly written as: $\mathbf{q}_{2}=\left(2 a^{*}+b^{*}+c^{*}\right) / 4.5$, which corresponds the mode of $\eta=2 / 9$.

Below the MT, we have observed a rich variety of domain structure. The micro-domain lamellas in most areas are very complex due to the evident structural distortion and defects induced by large strain appearing during the MT. $\mathrm{X}$-ray investigations indicate that the average structure of the low-temperature martensite has an orthorhombic unit cell [13]. Careful TEM observations suggest the resultant structural domains within the crystal planes, in which the structural modulations occur at low temperatures, could be very regular and clearly visible. Fig. 2(f) shows the typical domain structure within the (011)-crystal plane in a thin area. The average lamella space in this area is about $50 \mathrm{~nm}$. Detail structural features about low-temperature domains and their twining relationships will be reported in a forthcoming paper.

Premartensitic phenomena and systematic alternation of the structural modulations, in association with incomplete softening of the anomalous phonons, are also significant issues in the Heusler alloys. For instance, the ferromagnetic $\mathrm{Ni}-\mathrm{Mn}-\mathrm{Ga}$ undergoes a premartensitic transition driven by the magnetoelastic interaction $[11,12]$. In order to better understand the basic features of premartensitic state in Heusler alloy $\mathrm{Ni}_{2} \mathrm{FeGa}$, we have made a series of in situ observations on both $\mathbf{q}_{1}$ and $\mathbf{q}_{2}$ modulations in a large temperature range of 100-1100 K. Fig. 3(a) shows two series of electron diffraction patterns taken at different temperatures, illustrating the modifications of satellite reflections of $\mathbf{q}_{1}$ and $\mathbf{q}_{2}$ modulations, respectively. It is remarkable that the modulations show up systematical changes in wavelength, intensity and width $\Delta \mathbf{q}$ with the decrease in temperature. The satellite reflections at the temperatures above the Curie temperature $T_{\mathrm{c}}=430 \mathrm{~K}$ show up as weak diffuse streaks, these properties can be clearly recognized in the diffraction patterns taken at the high temperature of about $1000 \mathrm{~K}$. The superlattice reflections between $T_{\mathrm{c}}(430 \mathrm{~K})$ and $T_{\mathrm{m}}(145 \mathrm{~K})$ are diffuse spots in q-space. For instance, we found the widths $\Delta \mathbf{q}_{1} \cong 0.16 \AA^{-1}$ and $\Delta \mathbf{q}_{2} \cong 0.11 \AA^{-1}$ at room temperature. Fig. 3(b) and (c) shows the modulation vectors of $\mathbf{q}_{1}$ and $\mathbf{q}_{2}$ as a function of the temperature. In order to clearly illustrate the diffuse properties in the satellite reflections, we have shown the satellites spots in the elliptic shapes as obtained in experiments. It can be clearly recognized that both $\mathbf{q}_{1}$ and $\mathbf{q}_{2}$ have evident alternations in the pre-martensitic range, proposed to be driven by the magnetoelastic interaction. Below ferromagnetic transition $T_{\mathrm{c}}=430 \mathrm{~K}$, the wave
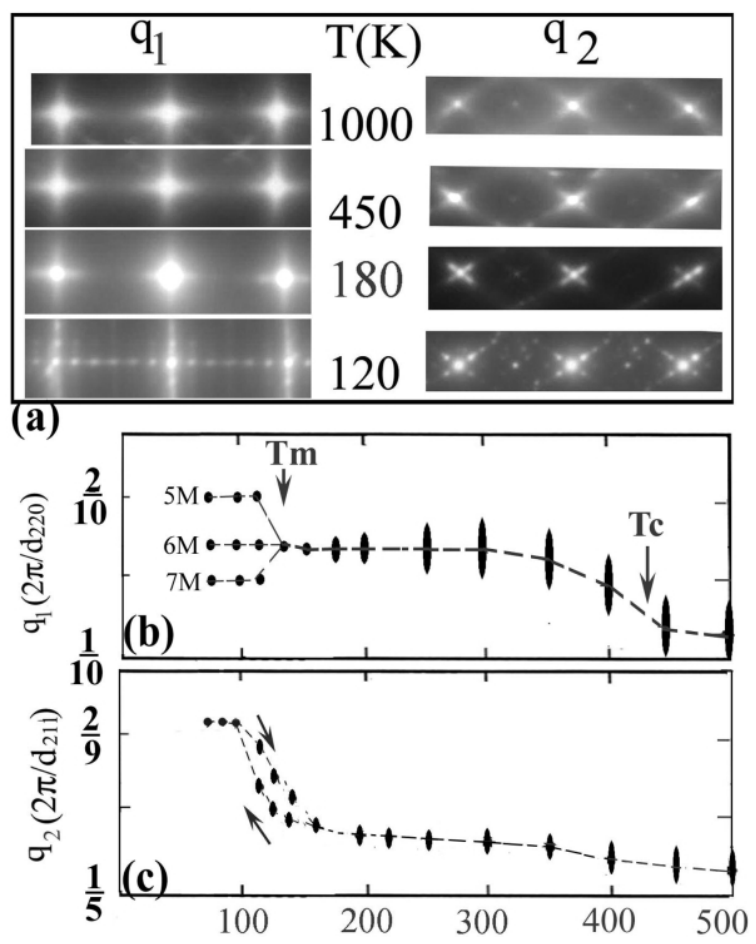

Fig. 3. (a) Electron-diffraction patterns showing the changes in satellite spots for both $\mathbf{q}_{1}$ and $\mathbf{q}_{2}$ modulations with lowering temperature. Temperature dependencies of (b) $\mathbf{q}_{1}$ and (c) $\mathbf{q}_{2}$ modulations, illustrating the systematical changes below ferromagnetic transition and evident anomalies around MT (145 K). 
vector of the $\mathbf{q}_{1}$ modulation first increase from $\zeta \approx 1 / 10$ $(1000 \mathrm{~K})$ to $\zeta \approx 1 / 3(330 \mathrm{~K})$ and then concentrate into weak spots with lowering temperature. In contrast with the $q_{1}$-modulation, $q_{2}$ shows only limited change following the ferromagnetic transition, its noticeable modification occurs just above the MT. At around $150 \mathrm{~K}$, the satellite streaks were quickly frozen at the systematical positions $(2 \eta \eta \eta) / 4.5$ as shown in Fig. 3(b). These results suggest that the magnetoelastic interaction is likely to yield different structural effects on the $[\zeta \zeta 0]$ and $[2 \eta \eta \eta]$ branches.

The other remarkable changes of modulations appear at the MT. The $\mathbf{q}_{1}$-modulation separates into three commensurate modes $5 \mathrm{M}, 6 \mathrm{M}$ and $7 \mathrm{M}$, as discussed in above context. The $\mathbf{q}_{2}$ modulation become into a very strong incommensurate one in association with complex lattice distortions, careful analyses indicate that the polarization of $\mathbf{q}_{2}$-modulation is roughly along the $\langle 0-11\rangle$ direction. The essential properties and the detailed atomic displacements for both $\mathbf{q}_{1}$ and $\mathbf{q}_{2}$ at low temperatures are still under investigation. In sharp contrast with the data observed at high temperatures, the satellite spots for either $\mathbf{q}_{1}$ or $\mathbf{q}_{2}$ become much more sharper below the MT, the sharpness of the satellite spots in the electron-diffraction patterns indicates the long coherent nature $(>200 \AA$ ) for these two modulations. Furthermore, we have carefully measured the hysteretic behaviors for both $\mathbf{q}_{1}$ and $\mathbf{q}_{2}$ modulation at the temperature of around MT, and showing the typical results of $\mathbf{q}_{2}$ in Fig. 3(c). Actually, the $\mathbf{q}_{1}$ modulation also shows up evident hysteretic features in correlation with the modulated-phase separation. This kind of hysteretic properties is complex and dependent sensitively on the local microstructure. A systematical structural investigation on this issue is in progress.

The anomalous features along with the MT have also been observed in the measurements of magnetization, resistivity, and strain [13]. The experimental results clearly revealed the discontinuous changes in correlation with the evident structural distortion resulting from this MT. In particular, our recent studies show that the magnetic structure has complex alternations during the MT. Fig. 4 shows the temperature dependence of the ac-susceptibility for a $\mathrm{Ni}_{2} \mathrm{FeGa}$ sample, clearly showing the presence of two jump-like turns at low temperatures. Careful structural

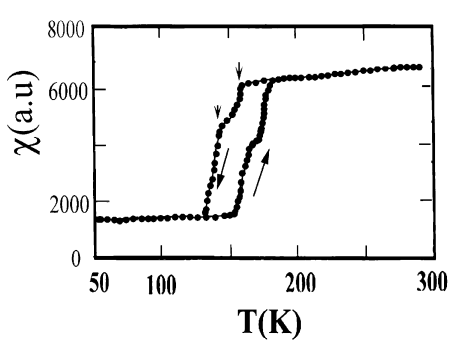

Fig. 4. Temperature dependence of ac magnetic susceptibility of a $\mathrm{Ni}_{2} \mathrm{FeGa}$ alloy, clearly displaying two jump-like turns during the MT. measurements suggest that the second step $(\sim 145 \mathrm{~K}$ in the cooling run) corresponds the appearance of martensite $5 \mathrm{M}$, $6 \mathrm{M}$, and $7 \mathrm{M}$ superstructures. The first step $(\sim 165 \mathrm{~K}$ in the cooling run) is likely to be attributed partially to other structural instabilities. We noted that two types of structural distortions corresponding to $\mathbf{q}_{1}$ and $\mathbf{q}_{2}$ develop in different ways during the MT. Hence, it is possible that the two steps revealed in the measurement of ac-susceptibility are, respectively, correlated with the structural amomalies along $\langle 110\rangle$ and $\langle 211\rangle$ directions. The other notable feature in the figure is the presence of hysteresis between the cooling and warming runs in the temperature range corresponding to the MT. Actually, similar behaviors, commonly appearing in other measurements, are believed to arise from the discontinuous structural modifications. It is also noted that the hysteresis loop of ac susceptibility commonly occurs slightly above MT, this phenomenon results possibly from the pre-martensitic transition, which could yield evident influence on the magnetic properties, and leads to the changes in intensity of the structural modulations $[5,7,11]$.

In summary, two distinctive structural modulations, along $\langle 110\rangle$ and $\langle 211\rangle$ directions, respectively, changes systematically along with the premartensitic and martensitic transitions in the Heusler alloy $\mathrm{Ni}_{2} \mathrm{FeGa}$ with $T_{\mathrm{m}}=145 \mathrm{~K}$. In the low-temperature martensite phase, the $\mathbf{q}_{1}$ modulation has a predominant mode at $(1 / 31 / 30)$, called $6 \mathrm{M}$ superstructure, and two additional modes called $5 \mathrm{M}$ and $7 \mathrm{M}$ superstructures, the $\mathbf{q}_{2}$ modulation show up as a strong incommensurate modulation. Certain anomalies in physical properties at low temperatures arise possibly from the structural alternations characterizing evolutions of the $\mathbf{q}_{1}$ and $\mathbf{q}_{2}$ modulations.

\section{Acknowledgements}

We would like to thank Professor Y.Q. Bei and Miss S.L. Jia for their assistance in preparing samples and measuring some physical properties. The work reported here was supported by the 'Hundreds of Talents' program organized by the Chinese Academy of Sciences, PR China, and by the 'Outstanding Youth Fund (J.Q. Li)' with Grant No. 10225415.

\section{References}

[1] J.A. Krumhansl, Y. Yamada, Mater. Sci. Engng A 127 (1990) 167.

[2] W. Petry, J. Phys. (France) IV, Colloq. 5 (1995) C2-15.

[3] A. Fujita, K. Fukamichi, F. Gejima, et al., Appl. Phys. Lett. 77 (2001) 3054.

[4] Y. Furuya, N.W. Hagood, H. Kimura, et al., Mater. Trans., JIM 39 (1998) 1248. 
[5] M. Wuttig, J. Li, C. Craciunescu, Scripta Materialia 44 (10) (2001) 2393.

[6] P.J. Webster, K.R.A. Ziebeck, S.L. Town, et al., Philos. Mag B 49 (1984) 295.

[7] R.D. James, M. Wuttig, Philos. Mag. A 77 (1998) 1273.

[8] G.H. Wu, C.H. Yu, L.Q. Meng, et al., Appl. Phys. Lett. 75 (1999) 2990

[9] R.C. O’Handley, J. Appl. Phys. 83 (1998) 3263.

[10] A. Sozinov, A.A. Likhachev, N. Lanska, et al., Appl. Phys. Lett. 80 (2002) 1746.
[11] A. Planes, E. Obrado, A. Gonzales-comas, et al., Phys. Rev. Lett. 79 (1997) 3926.

[12] A. Zheludev, S.M. Shapiro, P. Wochner, et al., Phys. Rev. B 51 (1995) 11310.

[13] Z.H. Liu, M. Zhang, Y.T. Cui, et al., Appl. Phys. Lett. (2003) in press.

[14] Y. Sutou, I. Ohnuma, R. Kainuma, et al., Metall. Mater. Trans. 29A (1998) 2225.

[15] V.V. Khovailo, T. Takagi, A.N. Vasilev, et al., Phys. Stat. Sol. (a) 183 (2001) R1. 